\title{
The copper-transporting capacity of ATP7A mutants associated with Menkes disease is ameliorated by COMMD1 as a result of improved protein expression
}

\author{
Willianne I. M. Vonk • Prim de Bie - Catharina G. K. Wichers • \\ Peter V. E. van den Berghe $\cdot$ Rozemarijn van der Plaats $\cdot$ Ruud Berger • \\ Cisca Wijmenga $\cdot$ Leo W. J. Klomp • Bart van de Sluis
}

Received: 20 December 2010 / Revised: 26 May 2011 / Accepted: 30 May 2011 / Published online: 11 June 2011

(C) The Author(s) 2011. This article is published with open access at Springerlink.com

\begin{abstract}
Menkes disease (MD) is an X-linked recessive disorder characterized by copper deficiency resulting in a diminished function of copper-dependent enzymes. Most MD patients die in early childhood, although mild forms of MD have also been described. A diversity of mutations in the gene encoding of the Golgi-resident copper-transporting $\mathrm{P}_{1 \mathrm{~B}}$-type ATPase ATP7A underlies MD. To elucidate the molecular consequences of the ATP7A mutations, various mutations in $A T P 7 A$ associated with distinct phenotypes of MD (L873R, C1000R, N1304S, and A1362D) were analyzed in detail. All mutants studied displayed
\end{abstract}

L. W. J. Klomp and B. van de Sluis contributed equally to this work.

Electronic supplementary material The online version of this article (doi:10.1007/s00018-011-0743-1) contains supplementary material, which is available to authorized users.

W. I. M. Vonk · P. de Bie · C. G. K. Wichers ·

P. V. E. van den Berghe - R. van der Plaats · R. Berger .

L. W. J. Klomp

Department of Metabolic and Endocrine Diseases,

Netherlands Metabolomics Center, University Medical Center

Utrecht, 3584 EA Utrecht, The Netherlands

W. I. M. Vonk · P. de Bie · C. Wijmenga

Complex Genetics Section, University Medical Center Utrecht, 3584 EA Utrecht, The Netherlands

Present Address:

P. de Bie

Vascular and Tumor Biology Research Center, The Rappaport

Faculty of Medicine and Research Institute, 31096 Haifa, Israel

Present Address:

P. V. E. van den Berghe

The Beatson Institute for Cancer Research, Garscube Estate,

Switchback Road, Bearsden, Glasgow G61 1BD, UK changes in protein expression and intracellular localization parallel to a dramatic decline in their copper-transporting capacity compared to ATP7A the wild-type. We restored these observed defects in ATP7A mutant proteins by culturing the cells at $30^{\circ} \mathrm{C}$, which improves the quality of protein folding, similar to that which as has recently has been demonstrated for misfolded ATP7B, a copper transporter homologous to ATP7A. Further, the effect of the canine copper toxicosis protein COMMD1 on ATP7A function was examined as COMMD1 has been shown to regulate the proteolysis of ATP7B proteins. Interestingly, in addition to adjusted growth temperature, binding of COMMD1 partially restored the expression, subcellular localization, and copper-exporting activities of the ATP7A mutants. However, no effect of pharmacological chaperones was observed. Together, the presented data might

\footnotetext{
C. Wijmenga

Department of Genetics, University Medical Center Groningen, University of Groningen, 9700 RB Groningen, The Netherlands

B. van de Sluis $(\square)$

Department of Pathology and Laboratory Medicine,

University Medical Center Groningen, University of Groningen,

9713 AV Groningen, The Netherlands

e-mail: a.j.a.van.de.sluis@med.umcg.nl
} 
provide a new direction for developing therapies to improve the residual exporting activity of unstable ATP7A mutant proteins, and suggests a potential role for COMMD1 in this process.

Keywords ATP7A - Copper - COMMD1 - Menkes disease $\cdot$ Pharmacological chaperones $\cdot$ Protein folding

\begin{tabular}{ll}
\multicolumn{2}{l}{ Abbreviations } \\
ATOX1 & Antioxidant protein 1 \\
ATP7A & ATP-dependent copper transporter 7A \\
ATP7B & ATP-dependent copper transporter 7B \\
ATP8B1 & P-type ATPase member 8B1 \\
BCS & Bathocuproinedisulfonic acid \\
COMMD1 & Copper metabolism murr1 containing \\
& domain 1 \\
DMSO & Dimethyl sulfoxide \\
ER & Endoplasmatic reticulum \\
EV & Empty vector \\
GST PD & GST pull-down \\
IB & Immunoblotting \\
MD & Menkes disease \\
MEF & Mouse embryonic fibroblast \\
MRE & Metal-responsive element \\
OHS & Occipital horn syndrome \\
4-PBA & 4-Phenyl butyric acid \\
PM & Plasma membrane \\
shRNA & Short-hairpin RNA \\
TfR & Transferrin receptor \\
TGN & Trans-Golgi network \\
TMAO & Trimethylamine $N$-oxide \\
TMD & Trans-membrane domain \\
WD & Wilson's disease \\
WT & Wild-type \\
&
\end{tabular}

\section{Introduction}

The transition metal copper is an essential trace element required for a range of cellular processes, including neurotransmitter synthesis, mitochondrial respiration, antioxidant defense, connective tissue formation, pigmentation, peptide amidation, and iron metabolism (reviewed by [1]). The significance of copper is dramatically illustrated by the X-linked recessive disorder Menkes disease (MD; OMIM \#309400), which is characterized by copper deficiency (reviewed by [2-4]). Based on the severity of the phenotype, three classes of MD can be distinguished. Classical MD patients die in early childhood and suffer from severe mental retardation accompanied by epileptic insults, growth retardation, hypothermia, hypopigmentation, laxity of skin and joints, and the typical "kinky" or steely hair [4]. Patients displaying less severe or even no neurological defects are classified as mild MD or occipital horn syndrome (OHS), in which the clinical features of OHS are primarily related to defects in connective tissue formation [4].

MD results from mutations in ATP7A, encoding a 165-kDa copper-transporting $\mathrm{P}_{1 \mathrm{~B}}$-type ATPase [5-10]. Although the copper uptake from the diet into enterocytes is not disturbed in patients with MD, dysfunction of ATP7A affects copper transport across the intestinal mucosa into the blood and across the blood-brain barrier, leading to copper deficiency in many tissues. ATP7A resides in the trans-Golgi network (TGN), where it mediates copper incorporation into copper-dependent enzymes such as tyrosinase, lysyl oxidase, and peptidyl$\alpha$-monooxygenase. Upon increasing copper concentrations, ATP7A translocates to the plasma membrane (PM) to facilitate cellular copper excretion $[2,11,12]$. ATP7A missense mutations within conserved regions of the $\mathrm{P}_{1 \mathrm{~B}^{-}}$ type ATPase family generally lead to the development of $\mathrm{MD}$, and are associated with dysregulation of ATP7A protein synthesis, stabilization, trafficking, intracellular localization, copper-transporting capacity, and posttranslation modifications (reviewed by [3]). Indeed, mutations in the DKTG-motif (Fig. 1a), containing a conserved aspartic acid residue indispensable for ATP7A activity, affect the copper-induced translocation of ATP7A from the TGN to the PM. A comparable phenotype is seen when the copper-binding CPC-motif (Fig. 1a), characteristic for heavy metal transporting P-type ATPases, is mutated. However, a clear correlation between mutations in ATP7A and MD phenotype has not yet been identified [10].

ATP7A is highly homologous to ATP7B, which is another member of the $\mathrm{P}_{1 \mathrm{~B}}$-type ATPase family. ATP7B is involved in copper export from hepatocytes into the bile [2, 3]. Mutations in $A T P 7 B$ are associated with the hepatic copper overload disorder Wilson's disease (WD; OMIM \#277900), and can lead to misfolded, unstable proteins that mislocalize and consequently are impaired in their coppertransporting function. Interestingly, these defects can be partially restored by adjusting growth temperature or by addition of pharmacological chaperones [13]. In addition, we have previously demonstrated a role for COMMD1, encoded by the canine copper toxicosis gene COMMD1, in ATP7B protein stability. We speculated that COMMD1 mediates the proteolysis of misfolded, newly synthesized ATP7B proteins [14], and thus might be involved in the pathogenesis of WD.

In the present study, we characterized the molecular consequences of a number of ATP7A mutations that are associated with distinct phenotypes of MD (L873R, C1000R, N1304S, and A1362D). We investigated whether 
A

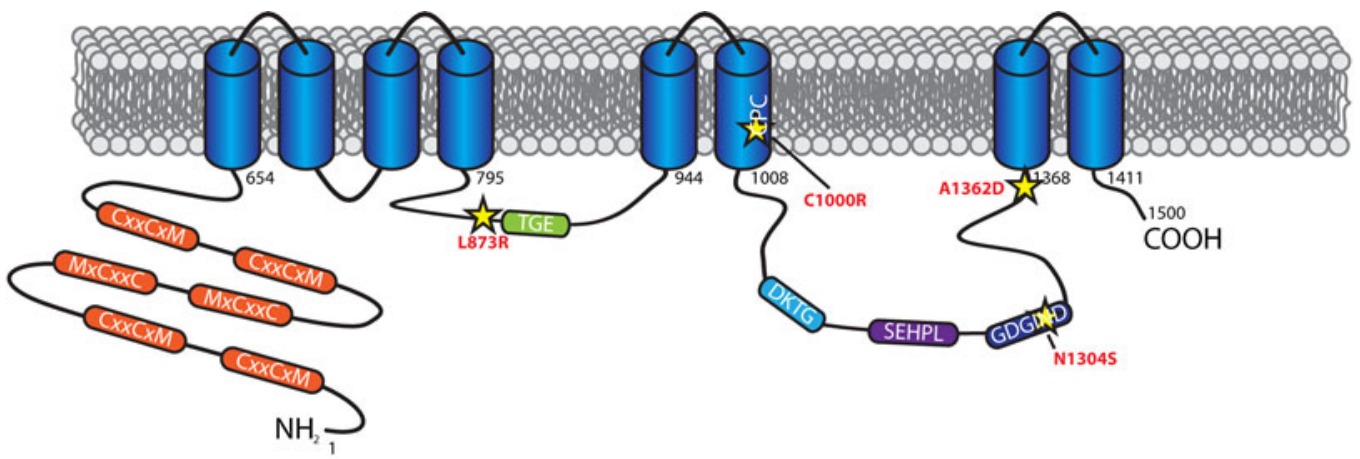

B
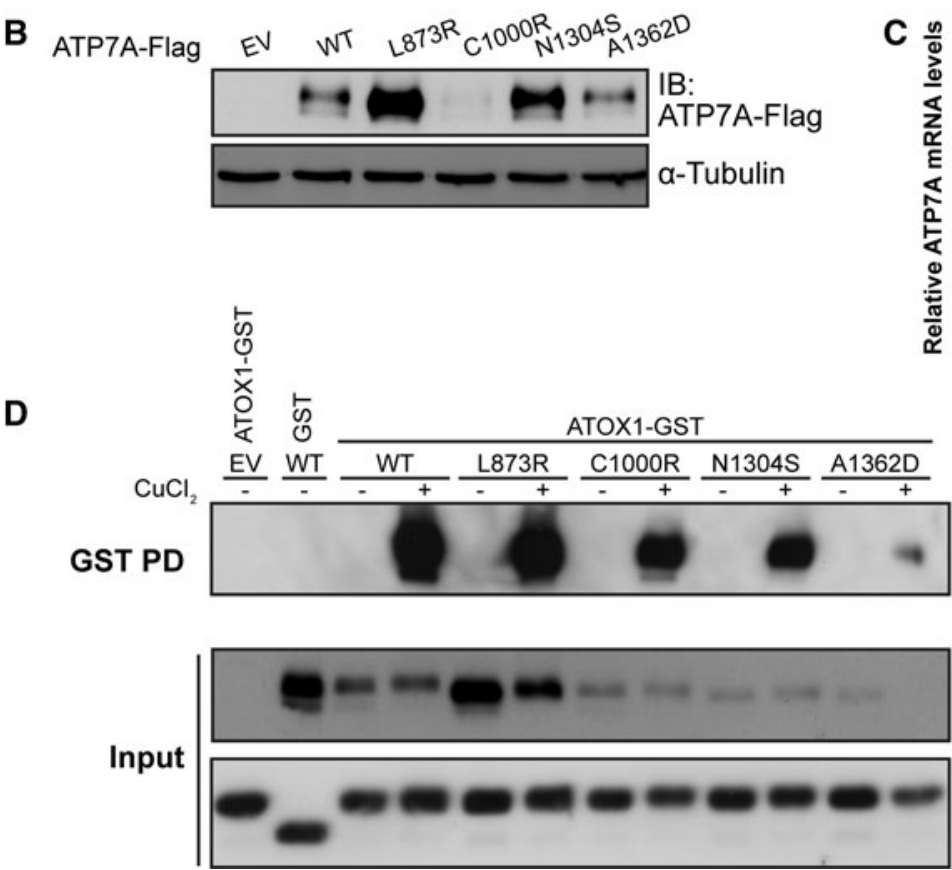

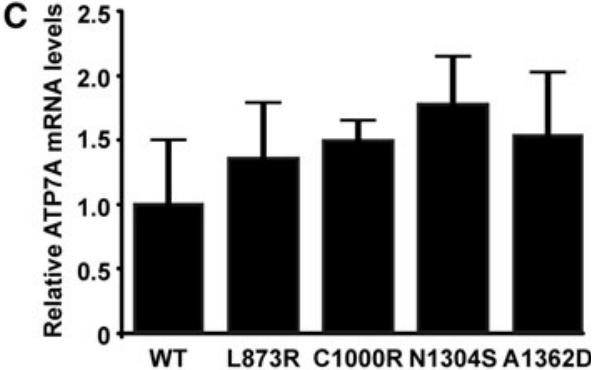

IB: ATP7A-Flag ATP7A-Flag ATOX1-GST GST
Fig. 1 Copper-dependent interaction of ATP7A mutant proteins with ATOX1 is unaffected despite their variations in protein expression. a Schematic representation of distinct functional domains in ATP7A. ATP7A contains eight trans-membrane spanning helices. At the $\mathrm{N}$-terminus, six metal binding sites (MxCxxC-motifs) are located where each can bind one $\mathrm{Cu}^{+}$ion. The nucleotide-binding domain, containing the conserved SEHPL-motif and DKTG-motif, is located in the cytosolic loop between TMD 6 and 7. After ATP binding and hydrolysis by the SEHPL-motif, the aspartic acid in the DKTG-motif accepts the phosphate group, resulting in the formation of a phosphorylated intermediated ATP7A protein. The actuator domain contains the TGE-motif, which is involved in dephosphorylation of the protein. The LL-motif at the end of the C-terminus regulates retrograde transport of the protein from vesicles or the plasma membrane (PM) to the trans-Golgi network (TGN). ATP7A mutations studied in this report are depicted with a yellow star. b HEK293T cells were transiently transfected with constructs encoding pcDNA (empty vector; EV), ATP7A-flag wild-type (WT) or mutant (L873R,
C1000R, 1304S, and A1362D) as indicated. Protein lysates (30 $\mu \mathrm{g})$ were analyzed by SDS-PAGE and immunoblotting (IB) using antibodies directed against the Flag-tag or $\alpha$-Tubulin as indicated on the right side of the figure. c mRNA abundance of ATP7A-Flag WT or mutants, expressed in HEK293T cells, was analyzed by means of qRT-PCR as described in "Materials and methods". The data represent mean $\pm \mathrm{SD}$ of triplicate measurements for each condition. The data were corrected for transfection efficiency and GAPDH expression, and normalized to WT. d HEK293T cells were transiently transfected with EV or with constructs encoding ATP7A-Flag WT and mutants, GST only or ATOX1-GST, as indicated above the figure. Cells were incubated overnight under basal conditions (-) or with $150 \mu \mathrm{M} \mathrm{CuCl}_{2}$. Cells were lysed (lysis buffer supplemented with $1 \mathrm{mM} \mathrm{CuCl}_{2}$ ) and GST fusion proteins were precipitated using glutathione Sepharose (GSH) beads. Precipitates (GST PD) or total cell lysates (Input; $30 \mu \mathrm{g}$ ) were analyzed by SDS-PAGE and IB using antibodies directed against the Flag-tag or GST defects in mutant protein expression, subcellular localization, and copper-transporting function as a result of the mutation can be improved, similar as was previously demonstrated for ATP7B. In addition, the effect of COMMD1 on the molecular function of ATP7A WT and mutants was studied. 


\section{Materials and methods}

\section{Reagents}

The following antibodies were used for immunoprecipitation and immunoblotting: rabbit-anti-COMMD1 antiserum [15], polyclonal mouse-anti-Flag M2 HRP conjugated (Sigma-Aldrich, St. Louis, MO, USA), polyclonal rabbit-anti-GST (Santa Cruz Biotechnology, Santa Cruz, CA, USA), polyclonal rabbit-anti-HA (SigmaAldrich), mouse-anti-transferrin receptor (Invitrogen Life Technologies Corporation, Carlsbad, CA, USA), goatanti-ATP7A (Santa Cruz), polyclonal mouse-anti- $\alpha$-tubulin (Sigma-Aldrich), and monoclonal mouse-anti- $\beta$-actin (Abcam, Cambridge, MA, USA). For immunofluorescence, Flag-epitopes were visualized using rabbit-antiFlag antibody (Sigma-Aldrich), and the trans-Golgi network (TGN) was labeled with mouse-anti-p230 antibody (BD Transduction Laboratories, San Diego, CA, USA). Secondary labeling was performed using Alexa Fluor ${ }^{\circledR}$ 488- and 568-conjugated antibodies (Molecular Probes, Eugene, OR, USA). Recombinant human lysyl oxidase homolog 2 was obtained from R\&D Systems, Inc. (Minneapolis, MN, USA).

A non-targeting pool of siRNAs (siControl) and siRNA pools targeting hCOMMD1 (siCOMMD1; targeting sequences have been described previously [16]) were purchased as ON-TARGET plus SMART pools from Dharmacon (Chicago, IL, USA).

\section{Cell culture and transfections}

Human embryonic kidney 293T cells (HEK293T), human osteosarcoma cells (U2OS) (both obtained from ATCC, Manassas, VA, USA) and mouse embryonic fibroblasts (MEFs) were cultured in high-glucose Dulbecco's modified Eagle's medium GlutaMAX ${ }^{\mathrm{TM}} \quad(4.5 \mathrm{~g} / 1 \quad$ D-Glucose and Pyruvate; Invitrogen Life Technologies Corporation) supplemented with $10 \%$ fetal bovine serum (FBS), L-glutamine, penicillin and streptomycin at $37^{\circ} \mathrm{C}$ in $5 \% \mathrm{CO}_{2}$. Monoclonal HEK293T cell lines, stably transfected with pSUPER-RETRO vector (shControl) or a plasmid encoding a short hairpin RNA (shRNA) targeting the COMMD1 mRNA sequence (shCOMMD1), were described previously [17] and maintained in HEK293T medium supplemented with $1 \mu \mathrm{g} / \mu \mathrm{l}$ puromycin dihydrochloride (Sigma-Aldrich). In various experiments, cells were incubated overnight with $10-150 \mu \mathrm{M} \mathrm{CuCl}_{2}$ (Sigma-Aldrich) or 10-200 $\mu \mathrm{M}$ bathocuproinedisulfonic acid (BCS; SigmaAldrich), respectively, prior to lysis or fixation.

MEFs were isolated from E14 embryos carrying loxP restriction sites around the first exon of Commd1. Loss of
Commd1 expression was accomplished as described previously [18].

Cells were transiently transfected by the calcium phosphate precipitation method as described elsewhere [19]. For cell surface biotinylation and immunofluorescence assays, cells were transfected with linear polyethylenimine (PEI; Polysciences, Warrington, PA, USA) at a 1:5 DNA:PEI ratio in serum-free medium. Transfection of siRNA ON-TARGETplus SMARTpools was performed by means of Lipofectamine RNAiMAX (Invitrogen Life Technologies Corporation), according to the manufacturer's protocol. Cells were harvested $48 \mathrm{~h}$ after transfection for biochemical analysis.

\section{Constructs}

pEBB-HA-COMMD1 and pEBB-COMMD1-GST constructs have been described previously [20]. pEBBCOMMD1-GST a.a. 1-118 and a.a. 119-190 deletion constructs were kindly provided by Dr. E. Burstein (Dallas, TX, USA) [21]. pcDNA4-ATP7A-Myc construct was obtained from Dr. A. Monaco (Oxford, UK) [22] in which the Flag-tag sequence was introduced in front of the Myc-tag by the Quickchange site-directed mutagenesis method (Stratagene, La Jolla, CA, US). ATP7A-Flag deletion constructs (a.a. 1-654, a.a. 795-94, a.a. 1,008-1,368, and a.a. 1,411-1,500) were subcloned into the pEBB-Flag vector. MD-associated mutations (L873R, C1000R, N1304S, and A1362D) were introduced in pcDNA4-ATP7A-Flag-Myc by the Quickchange sitedirected mutagenesis method (primer sequences available upon request). Sequences were verified by automated sequence analysis. The pG3L3-E1b-TATA-4MRE construct (MRE luciferase reporter) has been described previously [23], whereas the pRL-TK construct was obtained commercially from the Promega Corporation (Madison, WI, USA).

\section{Quantitative reverse transcriptase PCR (qRT-PCR)}

Total RNA was isolated from cells by means of TRIZOL ${ }^{\circledR}$ (Invitrogen Life Technologies Corporation). cDNA synthesis was performed using random hexamers and Super Script II reverse transcriptase (Invitrogen Life Technologies Corporation). mRNA expression of ATP7A was analyzed by quantitative PCR using Sybrgreen (Bio-Rad Laboratories Inc, Hercules, CA, USA) and the MyIQ real-time PCR cycler (Bio-Rad) using primers listed in Supplementary Table 1. Results were presented as fold induction, and normalized to the expression of GAPDH, which was selected as the most stable reference gene. Zeocin mRNA expression was used as a transfection marker. 
Metal-responsive element-luciferase copper biosensor assays

The bioavailability of cytosolic copper was measured by MRE-luciferase copper reporter assays, which were performed as described previously [13, 23]. Briefly, 20,000 HEK293T cells were seeded in 96-well plates and cotransfected with $35 \mathrm{ng}$ pG3L3-E1b-TATA-4MRE construct (MRE-luciferase reporter), $0.25 \mathrm{ng}$ or $\mathrm{pRL}-\mathrm{TK}$ vector and $0.4 \mathrm{ng}$ empty vector $(\mathrm{EV})$ or pcDNA4-ATP7A constructs as indicated. Cells were incubated overnight with indicated concentration of $\mathrm{CuCl}_{2}$, whereafter the cells were rinsed in PBS and harvested in passive lysis buffer (Promega) according to the manufacturer's protocol. Samples were analyzed by luminometry (Berthold Technologies, Bad Wildbad, Germany) using the DualLuciferase reporter system (Promega) for firefly luciferase activity and Renilla luciferase activity, according to the manufacturer's protocol. The RLU (relative light units) were calculated by dividing firefly luciferase measurements by Renilla luciferase measurements. All values were expressed as fold induction relative to ATP7A WT.

GST pull-down assays and immunoblot analysis

Precipitation of GST-tagged proteins by means of GSH-Sepharose beads was performed as described in [24]. In short, transiently transfected cells were rinsed once in PBS prior to lysis in lysis buffer A $(25 \mathrm{mM}$ HEPES; $\mathrm{pH}$ 7.9, $100 \mathrm{mM} \mathrm{NaCl}, 1 \mathrm{mM}$ NaEDTA, 1\% Triton X-100, $10 \%$ glycerol) supplemented with $1 \mathrm{mM} \mathrm{Na} \mathrm{VO}_{4}, 1 \mathrm{mM}$ PMSF, $10 \mathrm{mM}$ DTT, and protease inhibitors (Complete; Roche, Basle, Switzerland). For lysis of copper-treated or BCS-treated cells, lysis buffer A was supplemented with $1 \mathrm{mM} \mathrm{CuCl}_{2}$ or $\mathrm{BCS}$, respectively $[14,25]$. Protein concentrations were determined by Bradford Protein Assay (Bio-Rad). In all interaction studies, equal amounts of proteins in lysis buffer A were used for precipitation. Input samples represented approximately $1 \%$ of protein amounts used for GST-precipitation. Except for detection of ATP7A, protein lysates were boiled at $95^{\circ} \mathrm{C}$ prior to gel loading. In all experiments, SDS-PAGE was followed by protein transfer onto nitrocellulose membranes (Schleicher \& Schuell's-Hertogenbosch, the Netherlands) for immunoblot analysis.

\section{Cell surface biotinylation assays}

U2OS cells were used for cell surface biotinylation assays. Cells were incubated under basal conditions, or overnight with $50 \mu \mathrm{M} \mathrm{CuCl}_{2}$ as indicated in the experiment. Two days after transfection, proteins present on the cell surface were biotinylated as described in [26].
Lysyl oxidase activity assays

For lysyl oxidase activity assays, 20,000 HEK293T cells, stably transfected with either shControl or shCOMMD1, were seeded in 96-well plates. Upon overnight incubation with 10 and $50 \mu \mathrm{M} \mathrm{BCS}$, or 0,10 , and $50 \mu \mathrm{M} \mathrm{CuCl}_{2}$, the lysyl oxidase activity was measured according to the manufacturer's protocol using the Amplite ${ }^{\mathrm{TM}}$ Fluorimetric Lysyl Oxidase Assay Kit (AAT Bioquest, Inc., Sunnyvale, CA, USA), in which released $\mathrm{H}_{2} \mathrm{O}_{2}$ is detected using an Amplite $^{\text {TM }}$ HRP substrate. Obtained values were plotted against a lysyl oxidase standard curve.

Indirect immunofluorescence and confocal laserscanning microscopy

HEK293T cells were grown on coverslips pre-coated with poly-L-lysine (1:10 dilution, Sigma-Aldrich). Forty-eight hours after transfection, cells were washed once in icecold PBS and fixed using 4\% (w/v) paraformaldehyde for $20 \mathrm{~min}$ at $4^{\circ} \mathrm{C}$. Cells were quenched with $50 \mathrm{mM} \mathrm{NH}{ }_{4} \mathrm{Cl}$ for $5 \mathrm{~min}$ at room temperature following blocking and permeabilization using blocking buffer $(0.2 \%$ (w/v) BSA (Sigma-Aldrich), $0.1 \%$ (w/v) saponin (Sigma-Aldrich) in PBS) for $1 \mathrm{~h}$ at room temperature in the dark. Immunolabeling was performed in blocking buffer with the indicated primary antibodies for $1 \mathrm{~h}$. After washing three times, secondary labeling was performed with AffiniPure Alexa Fluor $^{\circledR} 488$ or 568 antibodies. DAPI staining was performed in the final wash steps preceding the mounting of coverslips in Fluorsave (VWR International Ltd, Leicestershire, UK). Images were acquired using an LSM710 Meta confocal microscope (Carl Zeiss, Jena, Germany) equipped with a $63 \mathrm{X} / 1.40$ N.A. Plan-Apochromat objective by sequential excitation at 408, 488, and $561 \mathrm{~nm}$.

\section{Statistical analysis}

Relative protein expressions and interaction strengths were quantified using densitometry (GS-700; Bio-Rad). The quantitative data in this paper are represented as mean \pm SD. Statistical evaluation was made using Student's $t$ test and differences were considered to be significant at $p<0.05$.

\section{Results}

Menkes disease-associated mutations in ATP7A result in diminished copper-transporting capacities

For this study, we selected mutations in $A T P 7 A$, which are associated with either classical MD (L873R), mild MD 
(C1000R and A1362D), or OHS (N1304S and A1362D) (Fig. 1a, yellow stars). Several features of these mutants have already been reported by MD patient studies and yeast studies (summarized in Table 1). However, the detailed molecular consequences of these mutations on ATP7A expression, intracellular localization, and function in eukaryotic cells remain to be determined. First, we determined the expression of the mutant ATP7A proteins relative to the ATP7A wild-type (WT). Transient transfection of HEK293T cells with ATP7A WT or mutant constructs resulted in variable protein expression. Whereas the expression of the L873R and N1304S mutants was more abundant in comparison to WT, a clear decrease in the protein expression of the C1000R and A1362D mutants was observed (Fig. 1b). Importantly, as shown in Fig. 1c, the differences in protein expression could not be ascribed to alterations in the mRNA expression of the mutants relative to ATP7A WT. Despite their altered protein expression, GST pull-down analysis showed that all ATP7A mutants were still able to interact with the copper chaperone ATOX1 in a copper-dependent manner, similar to that seen for WT protein (Fig. 1d).

Second, we examined whether the mutations affected the copper-induced translocation of ATP7A from the TGN to the PM using indirect immunofluorescence and cell surface biotinylation assays. As shown previously, colocalization of ATP7A WT protein with the TGN marker p230 demonstrated that ATP7A is localized to the TGN under basal conditions (Fig. 2a; upper frames). In excess copper, ATP7A WT was completely translocated to the PM (Fig. 2a; lower frames). Interestingly, all ATP7A mutant proteins showed an altered subcellular localization compared to WT (Fig. 2b). In line with previous findings [27], mutant L873R was found to be constitutively present at the PM. N1304S was localized to the cell periphery and the PM under both conditions. Similar to ATP7A WT, C1000R, and A1362D mutants localized to the TGN; however, upon increasing copper concentrations, all immunoreactivity remained restricted to TGN, and no staining at the PM could be observed, indicating that both proteins failed to translocate to the PM. The PM localization of ATP7A WT and the mutant proteins L873R and N1304S was confirmed by biotinylation assays in U2OS cells. Upon copper incubation, ATP7A WT expression at the PM was markedly enhanced (Fig. 2c). In agreement with the immunofluorescence data, we demonstrated that mutant L873R is constitutively present on the PM. Similar to ATP7A WT, biotinylation studies of mutant N1304S revealed a copper-induced PM expression, although its occurrence at the PM was already higher compared to WT protein at basal conditions. Interestingly, the re-localization of both mutants from the PM back to the TGN was significantly affected. Mutant L873R remained localized to the PM in conditions of copper depletion, whereas N1304S expression was most abundant in the cell periphery (Fig. 2d). This indicates that both mutants were drastically impaired in their retrograde trafficking to the TGN.

Third, we investigated whether the copper-transporting activity of the different ATP7A mutants was affected. For this, an established luciferase-based copper biosensor was employed, which has previously been used to characterize the activity of copper transporters, including ATP7B [13, 23]. The copper biosensor design is based on the transactivation of the metallothionein-1 promotor in response to bioavailable cytosolic copper. An increase in the cytosolic copper concentration results in activation of the metal responsive transcription factor 1 (MTF-1), and subsequently promotes the expression of firefly luciferase by binding of MFT-1 to MREs positioned upstream of the firefly luciferase open reading frame in a copper-dependent fashion. In this way, the MRE-luciferase reporter measures the bioavailable cytosolic copper. Upon increasing copper concentrations, empty vector (EV) transfected cells showed a clear and saturable induction of reporter activity (Fig. 3a, black line). In the presence of ATP7A WT, the reporter activity was markedly diminished, reflecting ATP7Adependent export of copper from the cell (Fig. 3a, dark blue line). As shown in Fig. 3a, the copper-transporting capacities of all the mutants were clearly impaired and showed almost a similar induction of the copper reporter activity upon copper stimulation as was seen for

Table 1 List of studied Menkes disease-associated ATP7A mutants and their characteristics

\begin{tabular}{|c|c|c|c|c|c|c|}
\hline \multirow[t]{2}{*}{ Mutation } & \multirow[t]{2}{*}{ Affected domain } & \multirow[t]{2}{*}{ Clinical phenotype } & \multicolumn{2}{|c|}{ Localization } & \multirow{2}{*}{$\begin{array}{l}\mathrm{Cu} \text { transporting activity } \\
\text { (yeast studies) }\end{array}$} & \multirow[t]{2}{*}{ References } \\
\hline & & & Basal & Excess $\mathrm{Cu}$ & & \\
\hline L873R & A-domain (LITGEA-motif) & Classical MD & $\mathrm{CP}, \mathrm{PM}$ & PM & No copper response & {$[27,40]$} \\
\hline C1000R & TMD6 (ㅁC-motif) & Mild MD & TGN & TGN & No copper response & {$[6,27,41-43]$} \\
\hline N1304S & P-domain (GDGIND-motif) & OHS & ND & ND & $\sim 33 \%$ compared to WT & [44] \\
\hline A1362D & TMD7 & OHS/mild MD & TGN & TGN & $\sim 17 \%$ compared to $\mathrm{WT}$ & {$[6,35]$} \\
\hline
\end{tabular}

A-domain Actuator domain, $P$-domain phosphorylation domain, $M D$ Menkes disease, $O H S$ occipital horn syndrome, $P M$ plasma membrane, $C P$ cell periphery, $T G N$ trans-Golgi network, $N D$ not determined 
Fig. 2 ATP7A mutants display an altered subcellular localization compared to the wild-type protein. a HEK293T cells were transfected with ATP7A-Flag WT, and incubated overnight under basal conditions or with $150 \mu \mathrm{M}$ $\mathrm{CuCl}_{2}$. Cells were subsequently analyzed by indirect confocal immunofluorescence using antibodies against the Flag-tag (green). TGN was visualized by using p230 antibodies (red) and nuclei were stained with DAPI (blue). Bars: $10 \mu \mathrm{m}$.

b Localization of ATP7A mutants was determined as described under Fig. 2a. Bars: $10 \mu \mathrm{m}$. c Cell surface abundance of ATP7A-Flag WT and mutants L873R and N1304S was assessed by cell surface biotinylation in U2OS cells. Cells were incubated overnight with $50 \mu \mathrm{M} \mathrm{CuCl}_{2}$. Biotinylated proteins were precipitated by NeutrAvidin agarose beads. Precipitates (biotinylation) and total cell lysates (input; $30 \mu \mathrm{g}$ ) were analyzed by IB using the antibodies indicated. Transferrin (TfR) expression was used as a positive loading control. WT, L873R, and N1304S were analyzed on separate membranes. d Retrograde trafficking of ATP7A mutants L873R and N1304S was analyzed as described under Fig. 2a. Cells were incubated overnight under basal conditions, with $150 \mu \mathrm{M} \mathrm{CuCl}_{2}$ or with $200 \mu \mathrm{M}$ BCS, respectively. Bars: $10 \mu \mathrm{m}$
A
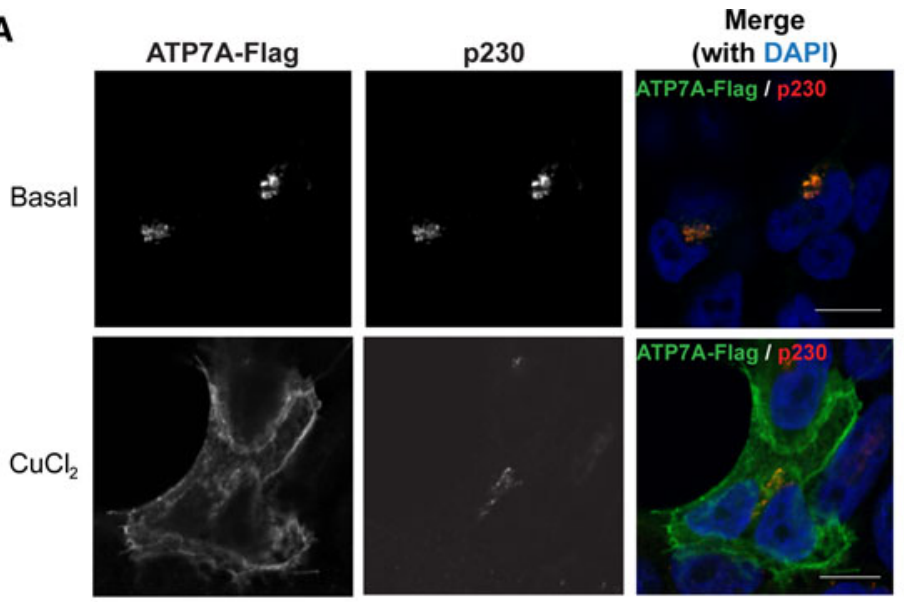

B
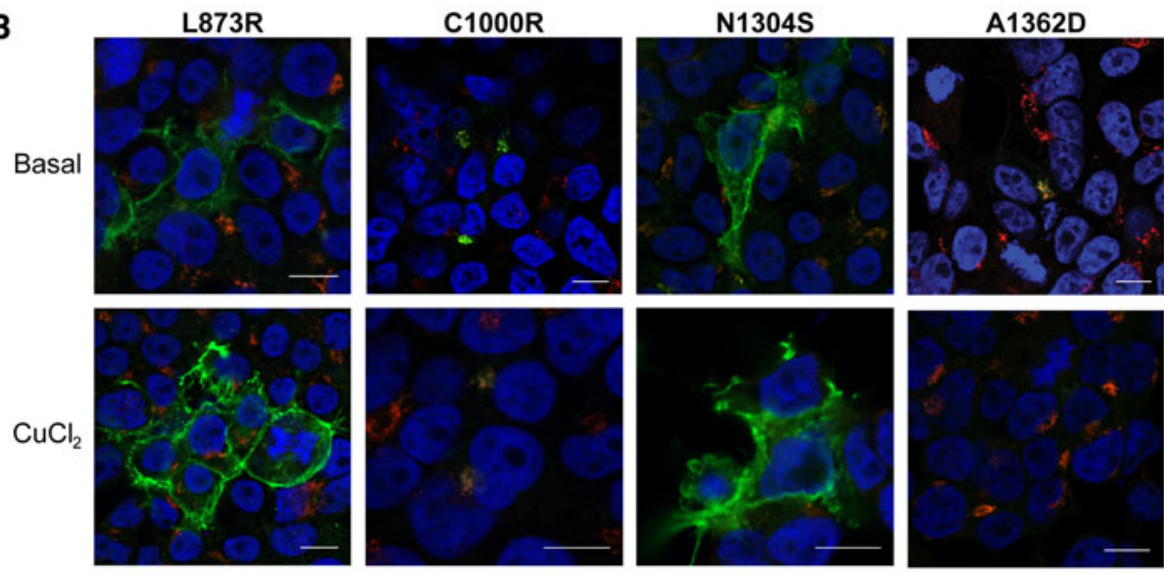

C
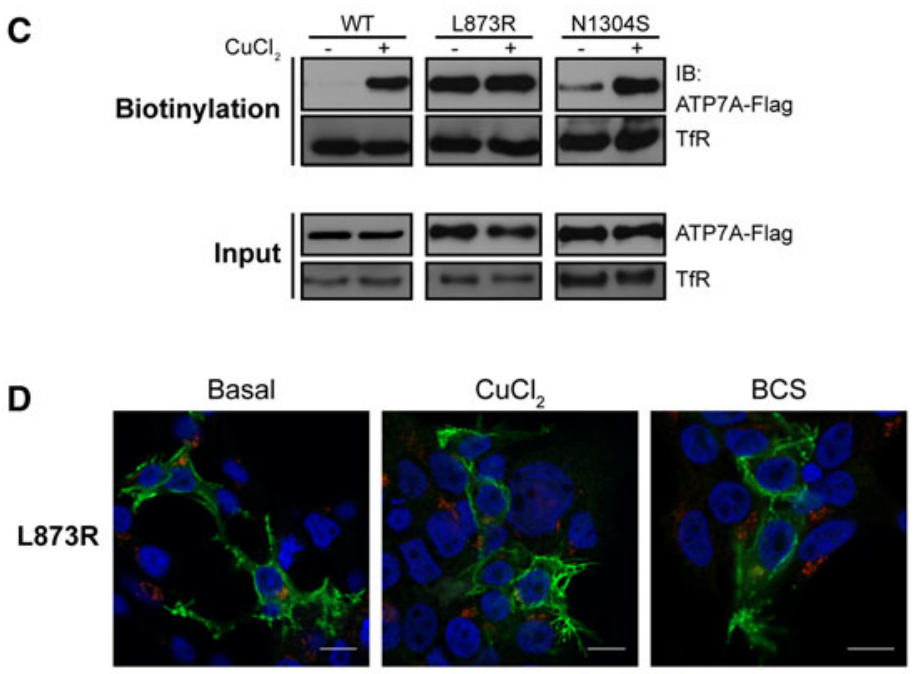

N1304S
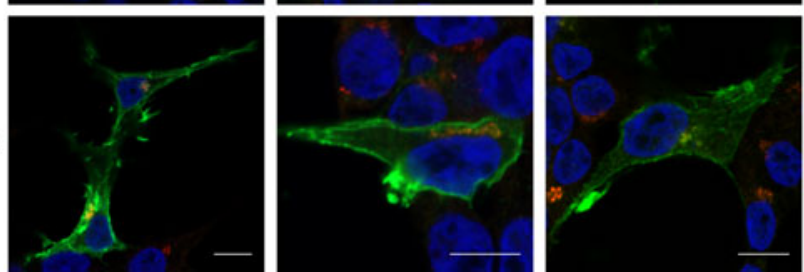
EV-transfected cells (Fig. 3a, b). These data indicate that all ATP7A mutations studied in this paper are associated with aberrant subcellular localization and for some mutations also with reduced protein expression. Consequently, these changes result in almost complete impairment of their copper-transporting capacity.

Mutant protein expression is impaired as a result of aberrant protein folding

In contrast to the enhanced expression of mutants L873R and N1304S, the protein level of mutants C1000R and A1362D was clearly reduced and is likely the result of increased proteolysis. To examine the potential role of
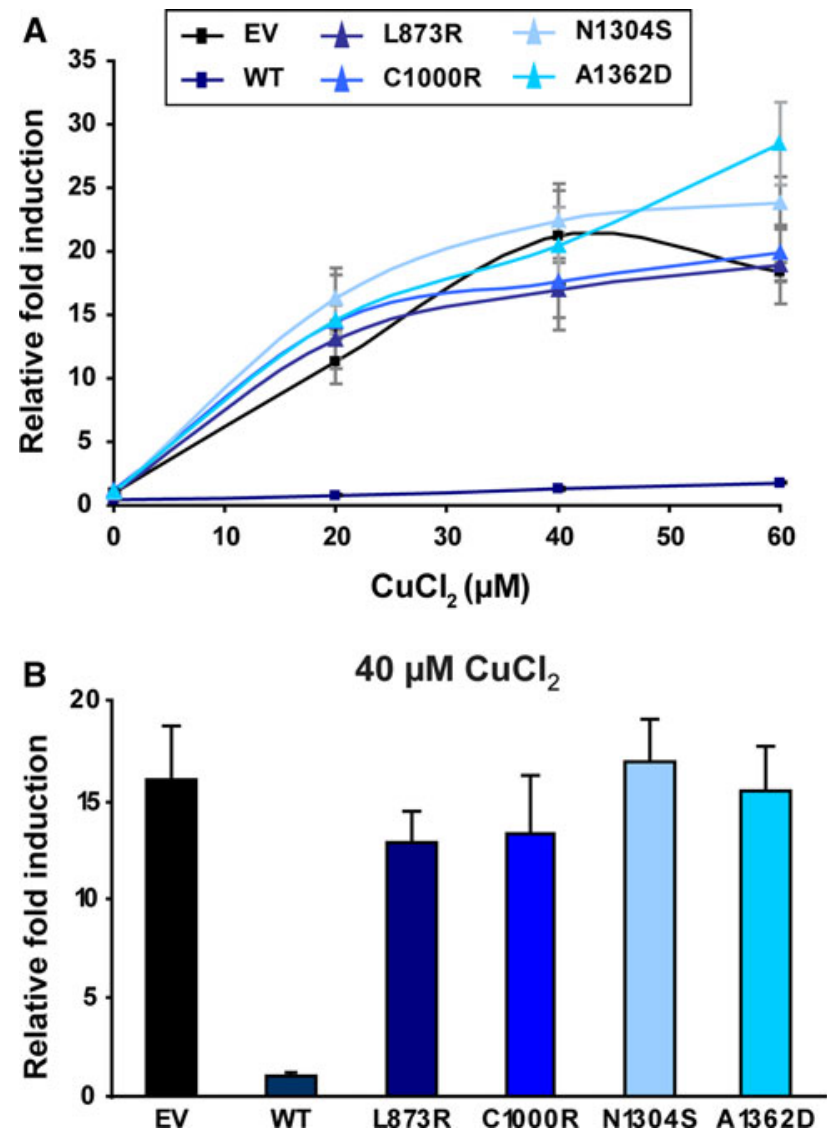

Fig. 3 Copper-transporting capacities of ATP7A mutants are drastically impaired compared to the wild-type. a HEK293T cells were transiently transfected with the MRE-luciferase copper reporter, together with EV (black line), ATP7A-Flag WT (dark blue line) or mutant constructs (light blue lines). After transfection, cells were incubated overnight with different concentrations of $\mathrm{CuCl}_{2}$ as indicated in the graph. MRE-luciferase copper reporter activities were measured and normalized for Renilla luciferase activity as transfection control. MRE-luciferase copper reporter activities are expressed as fold induction relative to WT. Values are $\pm \mathrm{SD}$. b Histogram describing relative fold induction of the copper reporter activity for EV and ATP7A WT and mutants as measured in Fig. 3a at $40 \mu \mathrm{M} \mathrm{CuCl}{ }_{2}$ proteasomal degradation on the decreased expression of C1000R and A1362D, cells were transfected with each of the mutants and incubated with the proteasomal inhibitor MG132. Blocking the proteasomal activity markedly improved the protein expression of both mutants (Fig. 4a), and the enhanced proteasomal degradation of C1000R and A1362D mutants might be the result of defective protein folding. To test this hypothesis, we improved the quality of the protein folding by reducing the growth temperature (as shown previously by $[13,26,28,29])$. HEK293T cells transiently transfected with either WT or ATP7A mutants C1000R and A1362D were grown at a low temperature $\left(30^{\circ} \mathrm{C}\right)$. At $30^{\circ} \mathrm{C}$, the expression of ATP7A WT, C1000R, and A1362D mutant proteins was markedly increased compared to cells cultured at $37^{\circ} \mathrm{C}$ (Fig. 4b). Next, we investigated the effects of the pharmacological folding chaperones 4-phenyl butyric acid (4-PBA) and curcumin on mutant protein expression. Incubation with 4-PBA or curcumin under conditions previously used to enhance the stability of other mutant P-type ATPases (e.g., ATP7B and ATP8B1) [13, 26], however, did not enhance the protein expression of ATP7A C1000R and A1362D (data not shown).

Since cellular growth at $30^{\circ} \mathrm{C}$ restores mutant protein expression, we determined whether protein localization and function can similarly be rescued by lowering the growth temperature. Although the subcellular localization of ATP7A WT protein was unaltered by a lower growth temperature, the intracellular localization of both mutants was clearly normalized. As shown in Figs. 2 and 4c, the expression of the $\mathrm{C} 1000 \mathrm{R}$ and A1362D mutants was mainly restricted to the TGN at $37^{\circ} \mathrm{C}$, whereas culture at $30^{\circ} \mathrm{C}$ induced a clear translocation to the cell periphery already at basal conditions (Fig. 4c). In excess copper, translocation to the PM of mutant A1362D was induced, similarly to ATP7A WT. Remarkably, copper reporter assays at this temperature demonstrated a significant decrease in the induction of the reporter to levels almost comparable to ATP7A WT, indicating a clear enhancement of the copper-transporting function of both ATP7A mutants (Fig. 4d). These data therefore suggest that the reduced copper-transporting capacities of the C1000R and A1362D mutants result from protein misfolding and subsequent proteasomal degradation or mislocalization.

COMMD1 binds ATP7A and improves the protein expression of ATP7A

Previously, we and others have demonstrated that COMMD1 regulates the proteolysis of a number of proteins, including ATP7B [14, 17, 30, 31]. To investigate whether ATP7A protein stability is also mediated by COMMD1, we studied the effect of COMMD1 on ATP7A expression. 
A

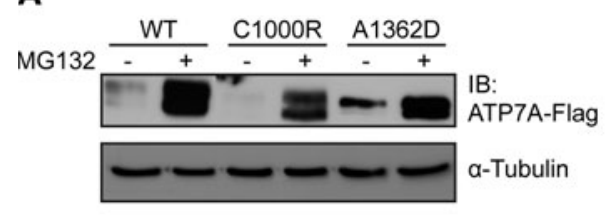

C

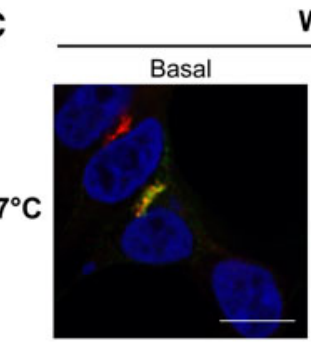

WT
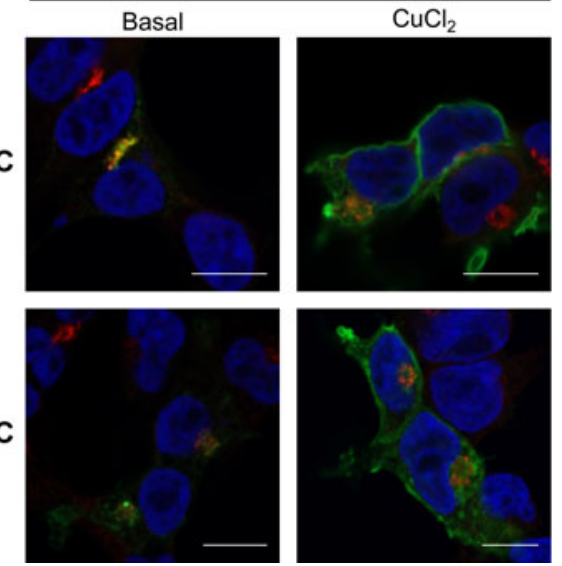

B

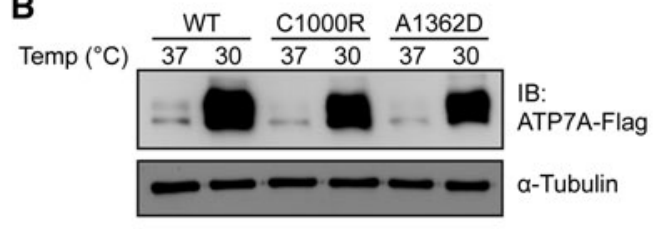

C1000R
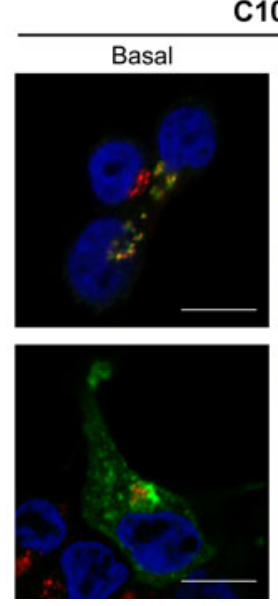

ery of ATP7A mutant protein expresFig. 4 Misfolding and recovery
sion. a HEK293T cells transiently transfected with ATP7A-Flag WT, and mutants $\mathrm{C} 1000 \mathrm{R}$ and $\mathrm{A} 1362 \mathrm{D}$ were treated overnight with DMSO or $3 \mu \mathrm{M}$ MG132. Cells were lysed and protein expression $(30 \mu \mathrm{g})$ was determined by IB. b HEK293T cells were transiently transfected with ATP7A-Flag WT, and mutants C1000R and $\mathrm{A} 1362 \mathrm{D}$, and cells were cultured for $48 \mathrm{~h}$ at 37 or $30^{\circ} \mathrm{C}$. Protein lysates $(30 \mu \mathrm{g})$ were subjected to Western-blot analysis using the antibodies indicated. c HEK293T cells were transfected with ATP7AFlag WT, and mutants C1000R and A1362D, and cultured at $30^{\circ} \mathrm{C}$.

Interestingly, overexpression of COMMD1 clearly enhanced the level of both ATP7A WT and mutant proteins (Fig. 5a). This effect of COMMD1 on ATP7A expression did not result from changes in ATP7A mRNA levels (Fig. 5b). In concordance, HEK293T cells stably transfected with shRNA targeting COMMD1 sequence (shCOMMD1) showed a decrease in ATP7A protein level (Fig. 5c), and ATP7A WT and all tested mutants were similarly affected by COMMD1 knockdown. These data were confirmed in HEK293T cells transiently transfected with siRNA targeting COMMD1 expression (Supplementary Fig. 1). In addition, shRNA-mediated downregulation of COMMD1 expression in HEK293T cells resulted in a significant decline in the protein level of endogenous ATP7A compared to control cells (Fig. 5d). Similar results were obtained in mouse embryonic fibroblasts (MEFs) lacking Commd1 expression (Fig. 5d).

Next, we elucidated to what extent COMMD1 expression affects the cellular copper homeostasis. In line with the observed decline in ATP7A protein level, downregulation of COMMD1 significantly induced the activity of the
Cells were incubated overnight under basal conditions or with $150 \mu \mathrm{M} \mathrm{CuCl} 2$. Cells were subsequently analyzed by indirect confocal immunofluorescence using antibodies against the Flag-tag (green) and p230 (red). Nuclei were stained with DAPI (blue). Bars: $10 \mu \mathrm{m}$. d Induction of the copper reporter was measured in HEK293T cells transiently transfected with the MRE-luciferase reporter, together with the ATP7A-Flag WT or mutant constructs C1000R and A1362D. Cells were cultured at $30^{\circ} \mathrm{C}$, and incubated overnight with $40 \mu \mathrm{M} \mathrm{CuCl}_{2}$. MRE-luciferase copper reporter activities are expressed as fold induction relative to WT. Values are $\pm \mathrm{SD}$

copper reporter. This suggests that copper efflux from the cell, mediated by ATP7A, is attenuated in COMMD1 depleted cells (Fig. 5e), but was complemented by exogenous ATP7A (Fig. 5e). To further corroborate the role of COMMD1 on ATP7A activity, we studied the biosynthesis of active cuproenzyme lysyl oxidase. Lysyl oxidase activity is dependent on the copper-transporting activity of ATP7A [32]. COMMD1 depletion resulted in significant reduction in lysyl oxidase activity in both HEK293T COMMD1 knockdown cells and in Commd1 KO MEFs compared to control cells (Fig. 5f).

Subsequently, we determined whether COMMD1 also interacts with ATP7A WT and mutant proteins, as has previously been demonstrated for ATP7B [13, 14]. GST PD analyses revealed a clear and specific interaction between COMMD1 and ATP7A WT (Fig. 6a). Although the expression of ATP7A WT and mutant proteins differed, we could not distinguish any obvious discrepancies in the binding of COMMD1 to ATP7A WT and mutants. Similar to what has been shown for all of the identified COMMD1 protein-protein interactions so far [14, 30, 31, 33, 34], the 
binding site for COMMD1 to ATP7A was mapped to the COMM domain of COMMD1 (a.a. residues 119-190; Fig. $6 \mathrm{~b}$ ), a sequence shared by all ten members of the COMMD protein family. ATP7A domains interacting with COMMD1 were restricted to the $\mathrm{N}$-terminus (a.a. 1-654) and to the cytosolic loop located between trans-membrane domain (TMD) 6 and 7 (a.a. 1,008-1,368) (Fig. 6c). Since the N-terminus of ATP7A is involved in copper binding, we determined whether the interaction between ATP7A and COMMD1 depended on copper. Upon excess copper or copper depletion by means of BCS, binding of ATP7A to COMMD1 was unaltered while the interaction with ATOX 1 could only be detected in conditions of excess copper, suggesting that COMMD1 binds to ATP7A in a copper-independent fashion (data not shown). Altogether, we here illustrate that COMMD1 expression modulates the protein level of both ATP7A WT and mutants, and subsequently cellular copper homeostasis.

COMMD1 ameliorates copper exporting function of ATP7A mutants

We continued to examine the effect of COMMD1 on the subcellular localization and copper-transporting capacity of ATP7A mutant proteins. Similar to the conditions for cellular growth at $30^{\circ} \mathrm{C}$, overexpression of COMMD1 induced a marked change in the localization of the mutants C1000R and A1362D from the TGN to the cell periphery and PM, respectively (Fig. 7). COMMD1 did not affect the localization of the L873R and N1304S mutants (data not shown). Additionally, no effect of COMMD1 on the cellular localization of ATP7A was established (Supplementary Fig. 2). Interestingly, as a consequence of improved protein expression and localization, COMMD1 ameliorated the copper exporting function of the ATP7A mutants as reflected by decreased induction of the copper reporter activity (Fig. 8a). Indeed, upon co-expression of COMMD1, a significant reduction in the copper reporter activity (approximately 29 and 36\%) was observed in cells expressing C1000R or N1304S mutant proteins, respectively (Fig. 8a). Exogenous COMMD1 in cells expressing mutants L873R and A1362D resulted in a trend towards an improved copper exporting capacity, although these changes were not significant. In line with these results, downregulation of COMMD1 expression led to a significant further increase in the induction of copper reporter activity for almost all ATP7A mutants (Fig. 8b).

\section{Discussion}

MD is caused by mutations in the ATP7A gene, however, the molecular consequence of these mutations is still
Fig. 5 COMMD1 improves ATP7A protein expression. a Effect of COMMD1 on ATP7A WT and mutant expression was studied in HEK293T cells expressing EV, ATP7A-Flag WT, or mutant proteins in combination with HA-COMMD1. Protein lysate $(30 \mu \mathrm{g})$ was analyzed by IB using indicated antibodies. $\mathbf{b}$ mRNA abundance of ATP7A-Flag WT or mutants, expressed in HEK293T cells, in absence of presence of HA-COMMD1 was analyzed by means of qRT-PCR as described in "Materials and methods". The data represent mean $\pm \mathrm{SD}$ of triplicate measurements for each condition. The data were corrected for transfection efficiency and GAPDH expression, and normalized to WT-EV. c HEK293T cells were stably transfected with pSUPER- RETRO vectors encoding non-specific shRNA (shControl) or shRNA against COMMD1 (shCOMMD1) together with ATP7A-Flag WT or mutant constructs. shRNA-mediated depletion of COMMD1 expression in HEK293T cells resulted in decreased protein expression of ATP7A-Flag WT and mutants visualized using immunoblot analysis (IB). Samples were analyzed on separate membranes (NT, WT, and L873R; and C1000R, N1304S, and A1362D). d ATP7A expression was analyzed in HEK293T cells upon shRNA-mediated depletion of COMMD1 expression (shControl vs. shCOMMD1) and in MEFs, isolated from wild-type mice (WT) and mice deficient for Commd1 (KO), by IB using the indicated antibodies. e Induction of the copper reporter was measured in HEK293T cells stably transfected with pSUPER- RETRO vectors encoding non-specific shRNA (shControl) or shRNA against COMMD1 (shCOMMD1) with indicated concentration ATP7A-flag-myc after overnight incubation with $40 \mu \mathrm{M} \mathrm{CuCl}_{2}$. NT non-transfected. MRE-luciferase copper reporter activities are expressed as fold induction relative to shControl. Values are \pm SD. * indicates a significantly different value compared to shControl cells $(* p<0.05)$. f Lysyl oxidase activity was determined in shControl and shCOMMD1 transfected HEK293T cells, and WT and Commd1 KO MEFs after overnight incubation with 10 and $50 \mu \mathrm{M}$ BCS or 0 , 10 , and $50 \mu \mathrm{M} \mathrm{CuCl}_{2}$. Values represent lysyl oxidase activity relative to shControl at basal conditions. Values are $\pm \mathrm{SD}$. *, **, and *** indicate significantly different values compared to shControl and COMMD1 KO cells, respectively $(* p<0.05$, **p $<0.01$, and $* * * p<0.005)$

unclear [5-10]. Here, we investigated four mutations in ATP7A associated with three distinct MD phenotypes; classical MD (severe MD), mild MD with long survival, and OHS. The characteristics of the selected ATP7A mutants on protein expression, intracellular localization, and copper transport were studied, and summarized in Table 2. Our most important observation was the clear improvement of the copper-transporting function of some of these ATP7A mutants in response to cellular growth at a low temperature $\left(30^{\circ} \mathrm{C}\right)$ and upon COMMD1 expression.

Two of the four mutations studied (C1000R and A1362D) resulted in decreased expression of the mutant ATP7A protein. This reduced expression was associated with enhanced proteasomal degradation rates, presumably as a result of protein misfolding. Indeed, improving protein folding by cellular growth at $30^{\circ} \mathrm{C}$ resulted in a significant increase in the protein expression of these mutants, and almost completely restored their copper transport activity. These findings are consistent with previous findings on other P-type ATPases, including ATP7B and ATP8B1 [13, 26]. Whereas misfolding of proteins is frequently 

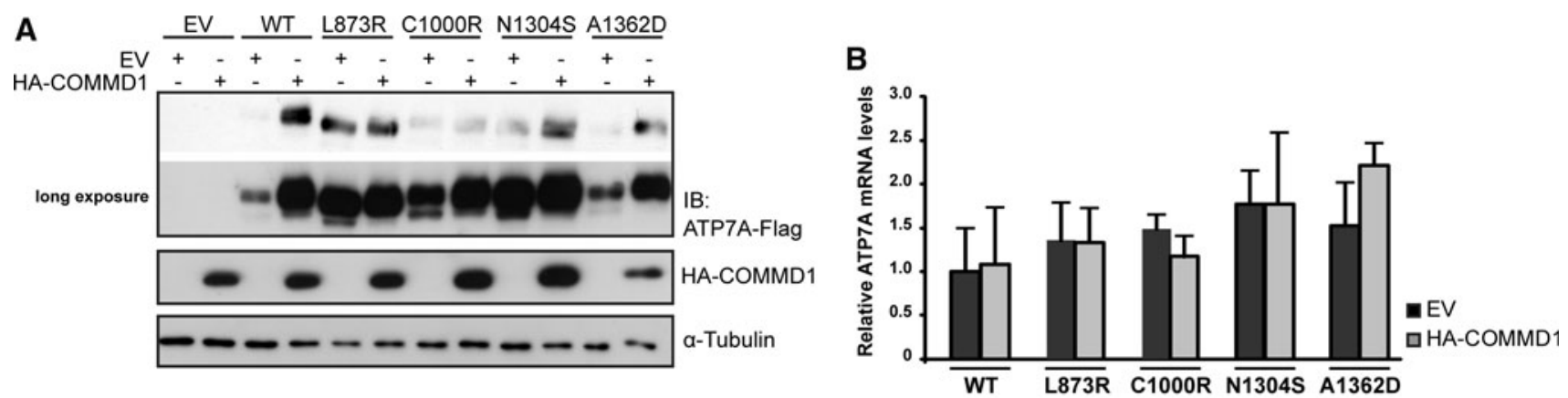

C
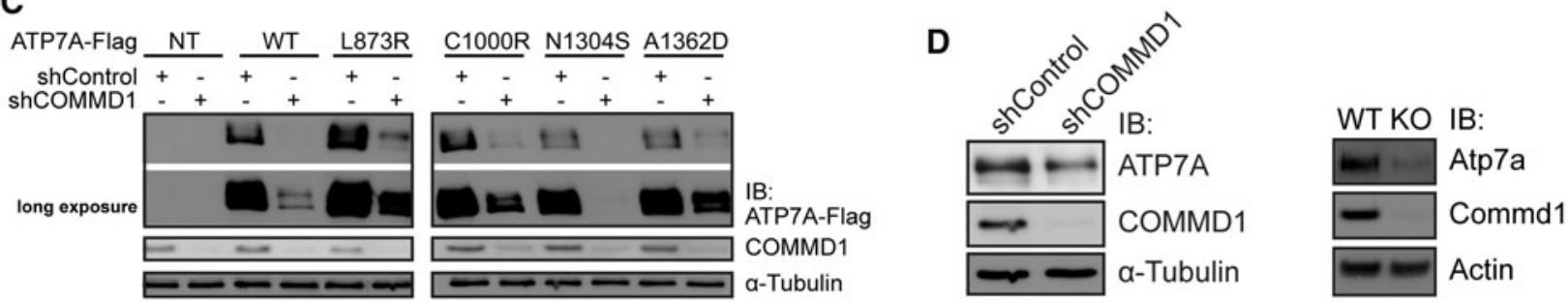

HEK293T

MEF
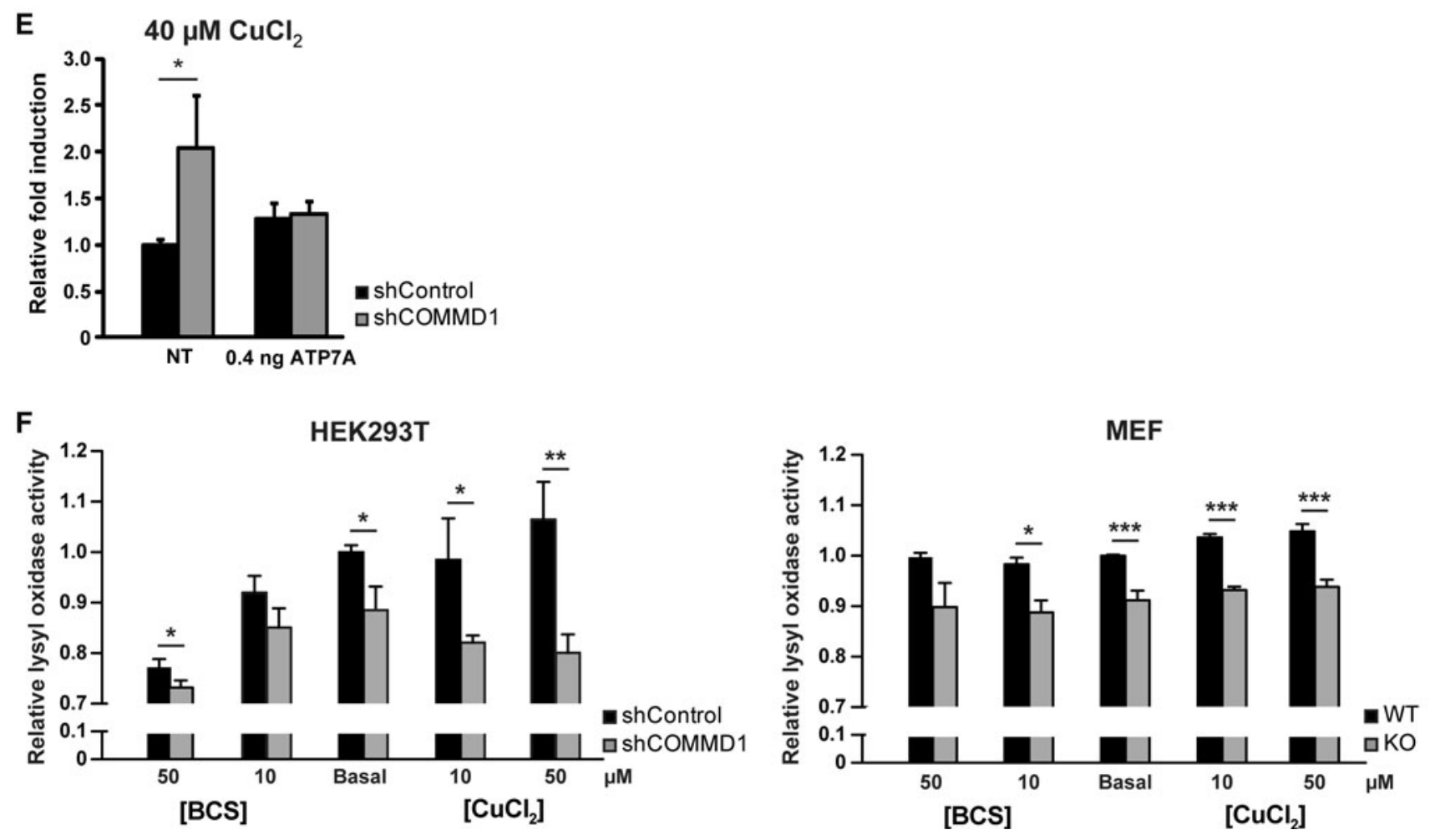

accompanied by aberrant intracellular localization, the ATP7A C1000R and A1362D mutants were properly localized to the TGN, but failed to translocate to the plasma membrane upon increased medium copper concentrations (previously shown for C1000R by [27]). In contrast to ATP7B and ATP8B1, no effect of the pharmacological chaperones 4-PBA and curcumin on ATP7A C1000R and A1362D mutant function was observed. Yet, similar to
4-PBA and curcumin, other compounds have been described as enhancing protein folding and stability (e.g., dimethyl sulfoxide (DMSO), trimethylamine $N$-oxide (TMAO), and glycerol), and therefore it might be interesting to evaluate their potential in improving ATP7A expression and function.

As a consequence of the mutations L873R and N1304S, associated with severe $\mathrm{MD}$ and $\mathrm{OHS}$, respectively, the 

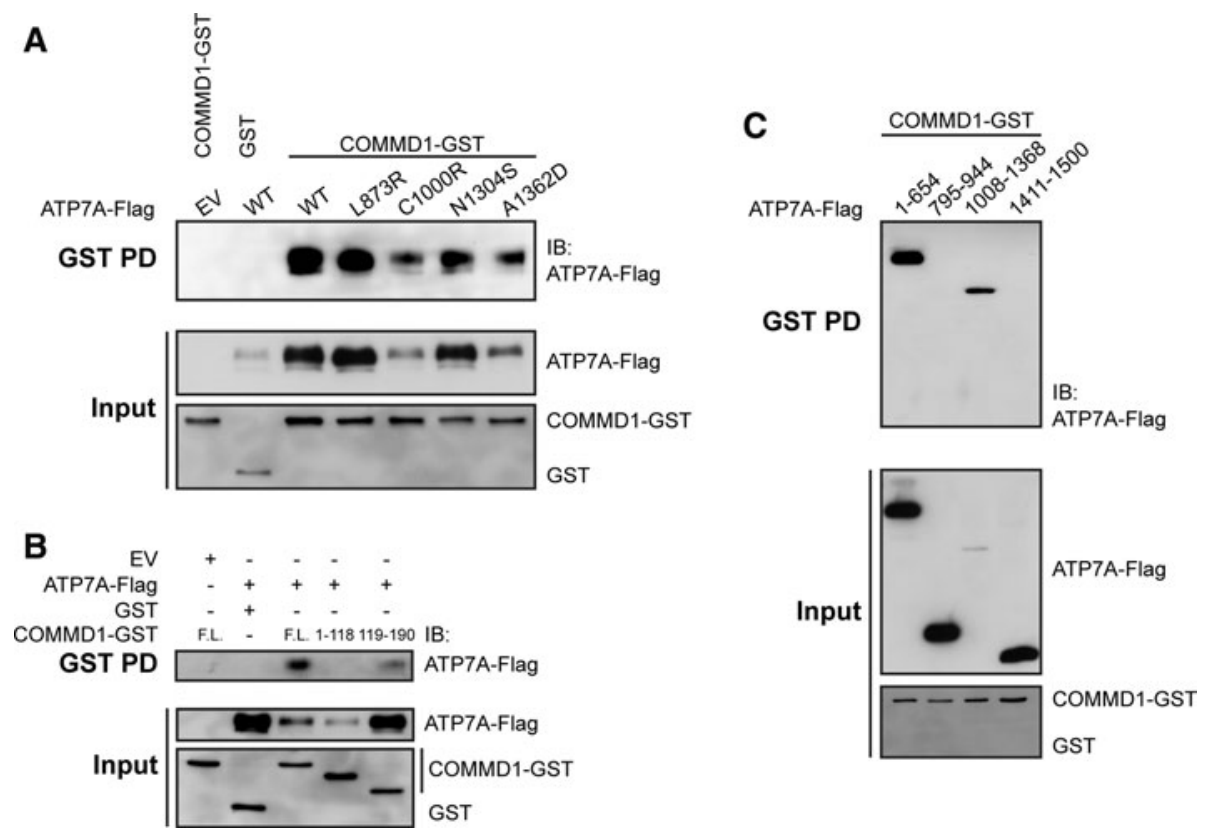

Fig. 6 ATP7A interacts with COMMD1. a HEK293T cells were transiently transfected with EV or with constructs encoding ATP7AFlag WT and mutants, GST only or COMMD1-GST as indicated above the figure. Cells were lysed and a GST pull-down assay was performed under basal conditions and analyzed as described in Fig. 1d. b HEK293T cells were transiently transfected with constructs encoding GST only, COMMD1-GST full-length (F.L.), N-terminal part (a.a. 1-118) or C-terminal region (COMM domain; a.a. 119-190)

in combination with ATP7A-Flag. Cells were lysed and a GST pulldown assay was performed and analyzed as described in Fig. 1d. c HEK293T cells were transiently transfected with constructs encoding COMMD1-GST and ATP7A-Flag N-terminal sequence (a.a. 1-164), internal loop TMD4-5 (a.a. 795-944), internal loop TMD6-7 (a.a. 1,008-1,368) or C-terminal region (a.a. 1,411-1,500). Cells were lysed, and a GST pull-down assay was performed and analyzed as described in Fig. 1d

EV

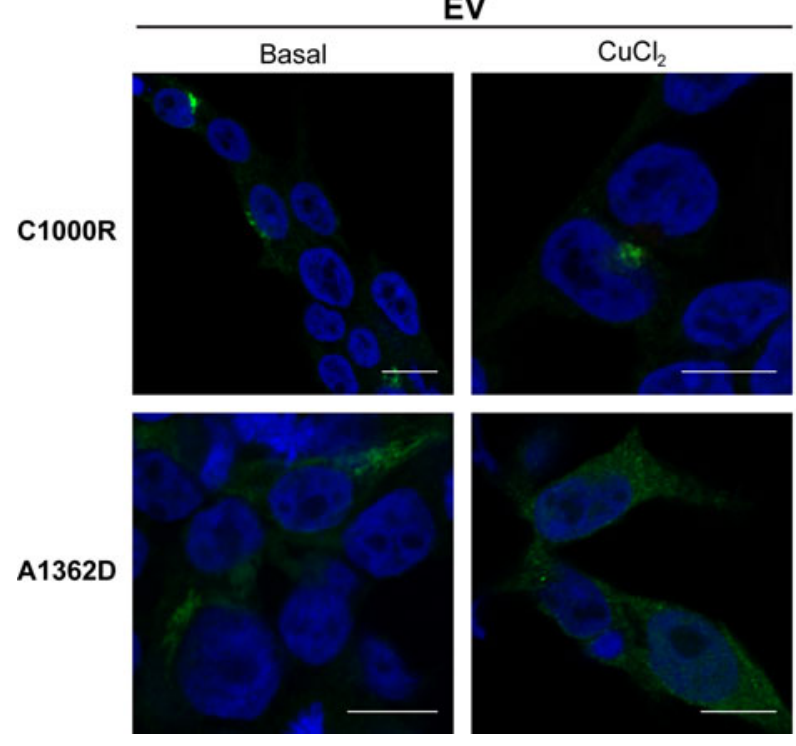

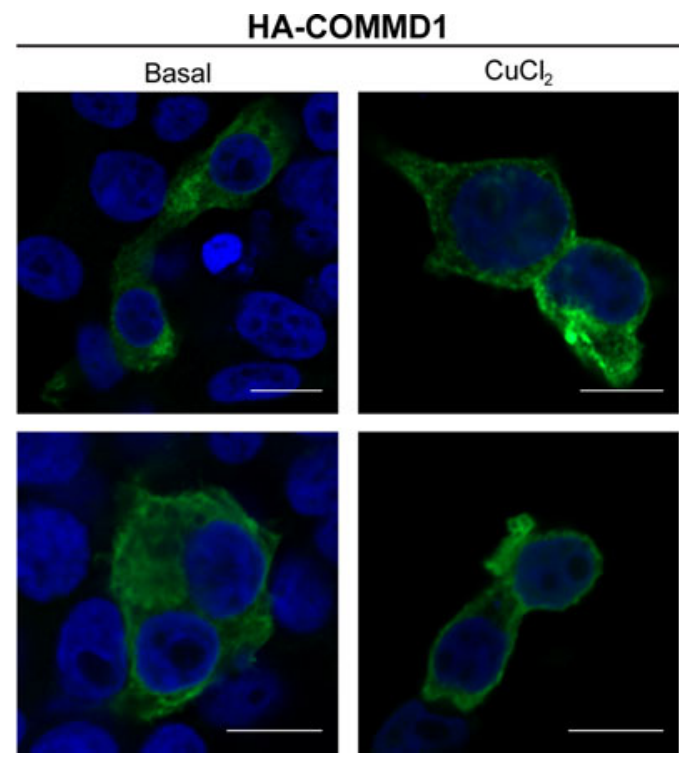

Fig. 7 Copper-induced trafficking of ATP7A mutants is largely restored by COMMD1. Localization of ATP7A mutants C1000R and A1362D was determined in HEK293T cells, transiently transfected with EV or HA-COMMD1. Cells were incubated overnight under

subcellular localization of these ATP7A mutant proteins was disturbed, and the copper export capacity was almost completely abolished. The expression of these mutant basal conditions or with $150 \mu \mathrm{M} \mathrm{CuCl} 2$. Cells were subsequently analyzed by indirect confocal immunofluorescence using antibodies against the Flag-tag (green). Nuclei were stained with DAPI (blue). Bars: $10 \mu \mathrm{m}$

proteins was, however, comparable to ATP7A WT or even enhanced. These results on mutant L873R are in line with the data recently published by Petris et al. [27]. Consistent 

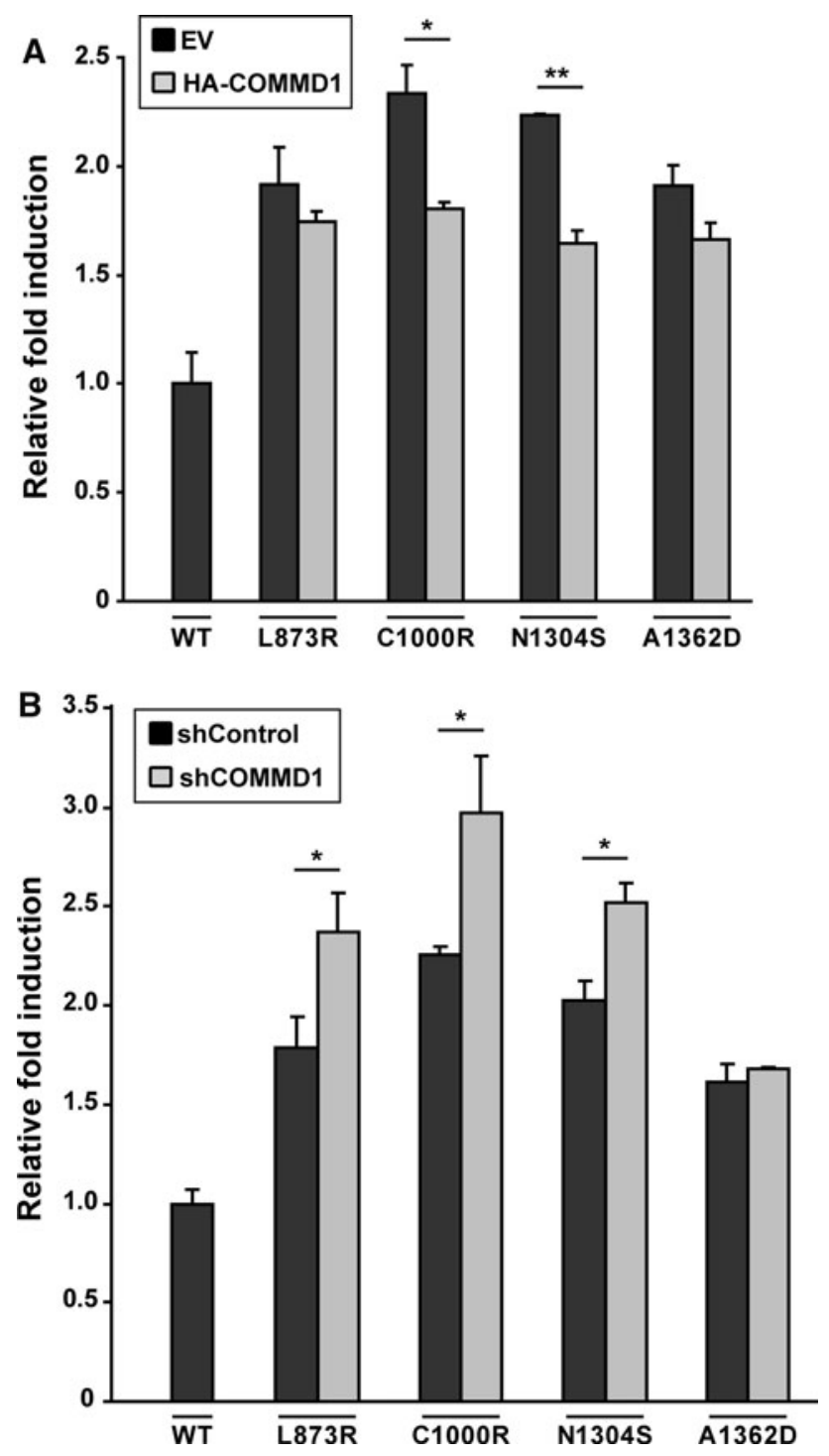

Fig. 8 COMMD1 partly ameliorates the copper-transporting function of ATP7A mutants. a Induction of copper reporter activity measured in HEK293T cells transiently transfected with the MRE-luciferase reporter in combination with ATP7A-Flag WT or mutant, EV and HA-COMMD1 constructs as indicated. Cells were incubated overnight with $40 \mu \mathrm{M} \mathrm{CuCl}_{2}$ concentrations. MRE-luciferase reporter activities are expressed as fold induction relative to WT. Values are $\pm \mathrm{SD}$. * and ** indicate significantly different values compared to EV transfected cells $(* p<0.05, * * p<0.005)$. b Induction of copper reporter was examined in HEK293T cells stably transfected with pSUPER- RETRO vectors encoding shControl or shCOMMD1, and the MRE-luciferase reporter in combination with ATP7A-Flag WT or mutant constructs as indicated. The assay was performed as described in Fig. 8a. * indicates significantly different values compared to shControl transfected cells $(* p<0.05)$

with the notion that these mutations are located in highly conserved functional domains essential for ATPase activity of the copper pump, we suggest that they may not induce aberrant folding, but rather result in a protein with severely affected or completely abolished copper-transporting activity. The aberrant expression of these proteins at the cell periphery under copper depleted conditions would explain the disrupted copper transfer into the secretory pathway for the biosynthesis of copper-dependent enzymes. However, in contrast to mutant L873R, N1304S mainly resides in the cell periphery and is to some extent also localized at the PM under copper-depleted or basal conditions. Additionally, in excess copper, this mutant shows a relatively normal copper response. These data suggest that this mutation does not completely abrogate the biosynthetic function of ATP7A, which correlates perfectly with its mild atypical Menkes disease phenotype. However, generally, it is currently still challenging to identify any genotype-phenotype correlation in MD patients, since a third of the mutations described are de novo, and there is inter- and intra-familial variability. For example, the A1362D mutation is associated with mild MD as well as with OHS in two different families [6,35]. In addition, the $\mathrm{R} 844 \mathrm{H}$ mutation has been identified in patients in one family with both a classical MD and mild MD phenotype $[6,36,37]$, whereas S637L and A1362V mutations are associated with all three MD phenotypes both within and between families [35, 38, 39]. The mechanisms behind these phenotypic variations are unknown, but they point to genetic and/or environmental factors that modulate disease severity.

Our data also demonstrate a novel role for COMMD1 in the protein expression and function of ATP7A mutant proteins. COMMD1 expression enhanced ATP7A levels, improved the subcellular localization, and ameliorated the copper exporting capacities of the ATP7A mutants. Since COMMD1 expression and cellular growth at $30^{\circ} \mathrm{C}$ both improved the molecular function of ATP7A mutants in an almost similar manner, we propose that COMMD1 might be involved in protein folding of ATP7A, and consequently in ATP7A stability, expression and function. Since decreased expression of COMMD1 clearly exacerbates the impaired function of ATP7A mutant proteins, it would be interesting to determine whether genetic variants of COMMD1 are associated with distinct MD phenotypes. We further evidently demonstrated that loss of COMMD1 expression attenuates ATP7A protein level and cellular copper homeostasis, indicating COMMD1 as novel modulator of ATP7A function.

In conclusion, in addition to mislocalization, our study clearly demonstrates that a number of mutations in ATP7A also display a reduced protein expression, most likely as a result of misfolding. Improving the protein folding ameliorates the copper exporting activity, and, depending on the molecular consequence of the mutation, might provide a new direction for therapeutic strategies to treat MD. Additionally, we also show a novel role for COMMD1 in regulating the copper-transporting function of ATP7A WT 
Table 2 Summary of systematic analysis of molecular consequences of mutations in ATP7A at 37 and $30^{\circ} \mathrm{C}$

\begin{tabular}{|c|c|c|c|c|c|c|c|c|c|c|c|c|c|c|c|}
\hline \multirow[t]{3}{*}{ ATP7A } & \multicolumn{4}{|c|}{ Expression $^{\mathrm{a}}$} & \multicolumn{2}{|c|}{ Interaction $^{\mathrm{b}}$} & \multicolumn{6}{|c|}{ Localization $^{\mathrm{c}}$} & \multicolumn{3}{|c|}{$\mathrm{Cu}$ transporting capacity ${ }^{\mathrm{d}}$} \\
\hline & \multirow[t]{2}{*}{$37^{\circ} \mathrm{C}$} & \multirow[t]{2}{*}{$30^{\circ} \mathrm{C}$} & \multirow[t]{2}{*}{ MG132 } & \multirow[t]{2}{*}{ COMMD1 } & \multirow[t]{2}{*}{ ATOX1 } & \multirow[t]{2}{*}{ COMMD1 } & \multicolumn{2}{|l|}{$37^{\circ} \mathrm{C}$} & \multicolumn{2}{|l|}{$30^{\circ} \mathrm{C}$} & \multicolumn{2}{|c|}{ COMMD1 } & \multirow[t]{2}{*}{$37^{\circ} \mathrm{C}$} & \multirow[t]{2}{*}{$30^{\circ} \mathrm{C}$} & \multirow[t]{2}{*}{ COMMD1 } \\
\hline & & & & & & & Basal & $\mathrm{Cu}$ & Basal & $\mathrm{Cu}$ & Basal & $\mathrm{Cu}$ & & & \\
\hline WT & + & +++ & +++ & ++ & + & + & TGN & $\mathrm{PM}$ & TGN & $\mathrm{PM}$ & TGN & PM & $100 \%$ & $100 \%$ & $100 \%$ \\
\hline L873R & +++ & ND & ND & +++ & + & + & $\mathrm{CP}, \mathrm{PM}$ & $\mathrm{PM}$ & ND & ND & $\begin{array}{l}\mathrm{CP}, \\
\mathrm{PM}\end{array}$ & $\mathrm{PM}$ & $\sim 0 \%$ & ND & NS \\
\hline C1000R & - & +++ & ++ & ++ & + & + & TGN & TGN & $\mathrm{CP}$ & $\mathrm{CP}$ & $\mathrm{CP}$ & $\mathrm{CP}$ & $\sim 0 \%$ & $\sim 80 \%$ & $\sim 30 \%$ \\
\hline N1304S & ++ & ND & ND & +++ & + & + & $\mathrm{CP}, \mathrm{PM}$ & $\mathrm{CP}, \mathrm{PM}$ & ND & ND & $\begin{array}{l}\text { CP, } \\
\text { PM }\end{array}$ & $\mathrm{CP}, \mathrm{PM}$ & $\sim 0 \%$ & ND & $\sim 35 \%$ \\
\hline A1362D & - & +++ & ++ & +++ & + & + & TGN & TGN, CP & $\begin{array}{l}\mathrm{CP}, \\
\mathrm{PM}\end{array}$ & $\begin{array}{l}\mathrm{CP}, \\
\mathrm{PM}\end{array}$ & $\begin{array}{l}\mathrm{CP}, \\
\quad \mathrm{PM}\end{array}$ & $\mathrm{CP}, \mathrm{PM}$ & $\sim 0 \%$ & $\sim 90 \%$ & NS \\
\hline
\end{tabular}

TGN Trans-Golgi network, $C P$ cell periphery, $P M$ plasma membrane, $N D$ not determined, $N S$ non-significant

${ }^{a}$ ATP7A mutant protein expressions are compared to WT expression (+) after incubation at $37,30^{\circ} \mathrm{C}$, after proteasomal inhibition by MG132 or by expression of COMMD1, respectively

${ }^{\mathrm{b}}$ Interactions of ATP7A mutants with ATOX1 and COMMD1 are compared to their interaction with ATP7A WT protein $(+)$

${ }^{c}$ Subcellular localization of ATP7A WT and mutant proteins in excess copper, at $37,30^{\circ} \mathrm{C}$, or by expression of COMMD1, respectively

${ }^{\mathrm{d}}$ Copper exporting capacities of mutant ATP7A proteins are relative to WT function (set at 100\%) and are based on the relative induction of the copper reporter at $37,30^{\circ} \mathrm{C}$ or after co-expression of COMMD1 (all in presence of $40 \mu \mathrm{M} \mathrm{CuCl2}$ )

and four ATP7A mutants associated with MD, and implicate COMMD1 as a potential modifier in MD pathogenesis.

Acknowledgments We thank Dr. E. Burstein and Dr. A. Monaco for kindly providing the COMMD1-deletion and pcDNA4-ATP7AMyc constructs, respectively. We are grateful to Dr. E. Burstein and Dr. H. Li for technical assistance with the MEFs experiments. We thank Jackie Senior for critical reading of the manuscript. This work was supported by the Netherlands Organization for Scientific Research (NWO, Grant number 40-00812-98-03106).

Open Access This article is distributed under the terms of the Creative Commons Attribution Noncommercial License which permits any noncommercial use, distribution, and reproduction in any medium, provided the original author(s) and source are credited.

\section{References}

1. Linder MC, Hazegh-Azam M (1996) Copper biochemistry and molecular biology. Am J Clin Nutr 63:797S-811S

2. La Fontaine S, Ackland ML, Mercer JF (2010) Mammalian copper-transporting P-type ATPases, ATP7A and ATP7B: emerging roles. Int J Biochem Cell Biol 42:206-209

3. de Bie P, Muller P, Wijmenga C, Klomp LW (2007) Molecular pathogenesis of Wilson and Menkes disease: correlation of mutations with molecular defects and disease phenotypes. J Med Genet 44:673-688

4. Tumer Z, Moller LB (2010) Menkes disease. Eur J Hum Genet 18:511-518

5. Tumer Z, Birk Moller L, Horn N (2003) Screening of 383 unrelated patients affected with Menkes disease and finding of 57 gross deletions in ATP7A. Hum Mutat 22:457-464
6. Moller LB, Bukrinsky JT, Molgaard A et al (2005) Identification and analysis of 21 novel disease-causing amino acid substitutions in the conserved part of ATP7A. Hum Mutat 26:84-93

7. Tumer Z, Moller LB, Horn N (1999) Mutation spectrum of ATP7A, the gene defective in Menkes disease. Adv Exp Med Biol 448:83-95

8. Gu YH, Kodama H, Murata Y et al (2001) ATP7A gene mutations in 16 patients with Menkes disease and a patient with occipital horn syndrome. Am J Med Genet 99:217-222

9. Das S, Levinson B, Whitney S, Vulpe C, Packman S, Gitschier J (1994) Diverse mutations in patients with Menkes disease often lead to exon skipping. Am J Hum Genet 55:883-889

10. Moller LB, Mogensen M, Horn N (2009) Molecular diagnosis of Menkes disease: genotype-phenotype correlation. Biochimie 91:1273-1277

11. Petris MJ, Mercer JF, Culvenor JG, Lockhart P, Gleeson PA, Camakaris J (1996) Ligand-regulated transport of the Menkes copper P-type ATPase efflux pump from the Golgi apparatus to the plasma membrane: a novel mechanism of regulated trafficking. EMBO J 15:6084-6095

12. Lonnerdal B (2008) Intestinal regulation of copper homeostasis: a developmental perspective. Am J Clin Nutr 88:846S-850S

13. van den Berghe PV, Stapelbroek JM, Krieger E et al (2009) Reduced expression of ATP7B affected by Wilson's diseasecausing mutations is rescued by pharmacological folding chaperones 4-phenylbutyrate and curcumin. Hepatology 50:1783-1795

14. de Bie P, van de Sluis B, Burstein E et al (2007) Distinct Wilson's disease mutations in ATP7B are associated with enhanced binding to COMMD1 and reduced stability of ATP7B. Gastroenterology 133:1316-1326

15. Klomp AE, van de Sluis B, Klomp LW, Wijmenga C (2003) The ubiquitously expressed MURR1 protein is absent in canine copper toxicosis. J Hepatol 39:703-709

16. Vonk WI, Wijmenga C, Berger R, van de Sluis B, Klomp LW (2010) $\mathrm{Cu}, \mathrm{Zn}$ superoxide dismutase maturation and activity are regulated by COMMD1. J Biol Chem 285:28991-29000

17. van de Sluis B, Muller P, Duran K et al (2007) Increased activity of hypoxia-inducible factor 1 is associated with early embryonic lethality in Commd1 null mice. Mol Cell Biol 27:4142-4156 
18. van de Sluis B, Mao X, Zhai Y et al (2010) COMMD1 disrupts HIF-1alpha/beta dimerization and inhibits human tumor cell invasion. J Clin Invest 120:2119-2130

19. Duckett CS, Gedrich RW, Gilfillan MC, Thompson CB (1997) Induction of nuclear factor kappaB by the CD30 receptor is mediated by TRAF1 and TRAF2. Mol Cell Biol 17:1535-1542

20. de Bie P, van de Sluis B, Burstein E et al (2006) Characterization of COMMD protein-protein interactions in NF-kappaB signalling. Biochem J 398:63-71

21. Maine GN, Mao X, Muller PA, Komarck CM, Klomp LW, Burstein E (2009) COMMD1 expression is controlled by critical residues that determine XIAP binding. Biochem J 417:601-609

22. Francis MJ, Jones EE, Levy ER, Ponnambalam S, Chelly J, Monaco AP (1998) A Golgi localization signal identified in the Menkes recombinant protein. Hum Mol Genet 7:1245-1252

23. van den Berghe PV, Folmer DE, Malingre HE et al (2007) Human copper transporter 2 is localized in late endosomes and lysosomes and facilitates cellular copper uptake. Biochem $\mathrm{J}$ 407:49-59

24. Burstein E, Ganesh L, Dick RD et al (2004) A novel role for XIAP in copper homeostasis through regulation of MURR1. EMBO J 23:244-254

25. Hamza I, Schaefer M, Klomp LW, Gitlin JD (1999) Interaction of the copper chaperone HAH1 with the Wilson's disease protein is essential for copper homeostasis. Proc Natl Acad Sci USA 96:13363-13368

26. van der Velden LM, Stapelbroek JM, Krieger E et al (2010) Folding defects in P-type ATP 8B1 associated with hereditary cholestasis are ameliorated by 4-phenylbutyrate. Hepatology 51:286-296

27. Petris MJ, Voskoboinik I, Cater M et al (2002) Copper-regulated trafficking of the Menkes disease copper ATPase is associated with formation of a phosphorylated catalytic intermediate. J Biol Chem 277:46736-46742

28. Wang X, Koulov AV, Kellner WA, Riordan JR, Balch WE (2008) Chemical and biological folding contribute to temperature-sensitive DeltaF508 CFTR trafficking. Traffic 9:1878-1893

29. Kim BE, Smith K, Meagher CK, Petris MJ (2002) A conditional mutation affecting localization of the Menkes disease copper ATPase. Suppression by copper supplementation. J Biol Chem 277:44079-44084

30. Maine GN, Mao X, Komarck CM, Burstein E (2007) COMMD1 promotes the ubiquitination of NF-kappaB subunits through a cullin-containing ubiquitin ligase. EMBO J 26:436-447

31. Thoms HC, Loveridge CJ, Simpson J et al (2010) Nucleolar targeting of RelA(p65) is regulated by COMMD1-dependent ubiquitination. Cancer Res 70:139-149
32. Royce PM, Camakaris J, Danks DM (1980) Reduced lysyl oxidase activity in skin fibroblasts from patients with Menkes' syndrome. Biochem J 192:579-586

33. van de Sluis B, Groot AJ, Vermeulen J et al (2009) COMMD1 promotes pVHL and $\mathrm{O} 2$-independent proteolysis of HIF-1alpha via HSP90/70. PLoS One 4:e7332

34. van de Sluis B, Groot AJ, Wijmenga C, Vooijs M, Klomp LW (2007) COMMD1: a novel protein involved in the proteolysis of proteins. Cell Cycle 6:2091-2098

35. Donsante A, Tang J, Godwin SC et al (2007) Differences in ATP7A gene expression underlie intrafamilial variability in Menkes disease/occipital horn syndrome. J Med Genet 44:492-497

36. Sztriha L, Janaky M, Kiss J, Buga K (1994) Electrophysiological and 99mTc-HMPAO-SPECT studies in Menkes disease. Brain Dev 16:224-228

37. Gerdes AM, Tonnesen T, Pergament E et al (1988) Variability in clinical expression of Menkes syndrome. Eur J Pediatr 148:132-135

38. Ambrosini L, Mercer JF (1999) Defective copper-induced trafficking and localization of the Menkes protein in patients with mild and copper-treated classical Menkes disease. Hum Mol Genet 8:1547-1555

39. Ronce N, Moizard MP, Robb L, Toutain A, Villard L, Moraine C (1997) A C2055T transition in exon 8 of the ATP7A gene is associated with exon skipping in an occipital horn syndrome family. Am J Hum Genet 61:233-238

40. Ogawa A, Yamamoto S, Takayanagi M, Kogo T, Kanazawa M, Kohno Y (1999) Identification of three novel mutations in the MNK gene in three unrelated Japanese patients with classical Menkes disease. J Hum Genet 44:206-209

41. Kim BE, Smith K, Petris MJ (2003) A copper treatable Menkes disease mutation associated with defective trafficking of a functional Menkes copper ATPase. J Med Genet 40:290-295

42. Lowe J, Vieyra A, Catty P, Guillain F, Mintz E, Cuillel M (2004) A mutational study in the transmembrane domain of Ccc2p, the yeast $\mathrm{Cu}(\mathrm{I})$-ATPase, shows different roles for each Cys-Pro-Cys cysteine. J Biol Chem 279:25986-25994

43. Bissig KD, Wunderli-Ye H, Duda PW, Solioz M (2001) Structure-function analysis of purified Enterococcus hirae CopB copper ATPase: effect of Menkes/Wilson's disease mutation homologues. Biochem J 357:217-223

44. Tang J, Robertson S, Lem KE, Godwin SC, Kaler SG (2006) Functional copper transport explains neurologic sparing in occipital horn syndrome. Genet Med 8:711-718 